\begin{tabular}{ccc}
\hline & International Journal of Engineering \& Technology, $7(2.12)(2018)$ 193-197 \\
SPC & International Journal of Engineering \& Technology \\
Website: $w$ ww.sciencepubco.com/index.php/IJET & Research Paper \\
\hline
\end{tabular}

\title{
Zadoff-chu sequence based preamble design of fbmc/oqam for $5 \mathrm{~g}$
}

\author{
Vamshi Krishna Namani ${ }^{1}$, R. Arthi ${ }^{2}$ \\ ${ }^{1}$ P.G Student,Department of Electronics and Communication Engineering \\ ${ }^{2}$ Professor, Department of Electronics and Communication EngineeringVignana Bharathi Institute of Technology, \\ Ghatkesar, Hyderabad - 501301, India \\ *Corresponding author E-mail: darthi73@gmail.com
}

\begin{abstract}
The Spectrum effectiveness for 5G networks can be accomplished by Filter Bank Multi Carrier / Orthogonal Quadrature Amplitude Modulation (FBMC / OQAM) that avoids the benefit of guard band. FBMC is very considerate to Carrier Frequency Offset (CFO) and Timing Offset (TO). This paper suggests Zadoff-chu (ZC) sequence based preamble or pilot is accustomed to design the FBMC frame for synchronize more with receiver to achieve sufficient channel inference and phase tracking which reduces the carrier frequency and timing synchronization. This method helps to influence the out of band emissions and conceive non-orthogonal waveforms to investigate the overall system performance such as Bit Error Rate (BER) over AWGN channels. The performance measuresbeing Complementary Cumulative Distribution Curve (CCDF) for FBMC signal with different multicarrier to evaluate the Peak to Average Power Ratio (PAPR) has been achieved by simulation. The simulation outcomeshows that FBMC outperforms well when analyzed with other multicarrier systems.
\end{abstract}

Keywords: FBMC; CFO; TO; BER; CCDF; PAP

\section{Introduction}

OFDM has a basicproblem [1] making it unattractive for forthcoming wireless communication services unattractive. The demerits of OFDM scheme are its large out - of - band emissions (OOBE), which affects the spectrum utilization, Doppler frequency shift, BER and large PAPR. In order for OFDM scheme to appropriate the strict requirements of time and frequency synchronization, guard interval, guard band to reduce OOBE in the real-world applications at the value of spectral and/or power efficiency, larger PAPR values and reduced bit error rate (BER) occurrence are studied. Fifth generation $(5 \mathrm{G})$ radio access technology has beenpredicted to take a huge advantages over earlier radio generations[2] by promoting cognitive radio and machine type communication. Different Access techniques [3] for 5G are Filter Bank Multicarrier (FBMC), Universal Filter Multicarrier (UFMC), and Generalized FrequencyDivision Multiplexing (GFDM) [4]. Among these techniques, FBMC was more suitable waveform contender for $5 \mathrm{G}$ because of its high spectrum utilization and shortened out of band emissions. Though FBMC has the drawback of CFO and TO synchronization, but this system was very conscious towards carrier parameters by receiver side.

Frequency localized prototype filters [5] like the PHYDYAS filters, with linear multi-tap equalizers and BER performances are better for FBMC compared with OFDM and with other $5 \mathrm{G}$ radio access technologies. Meanwhile, this access method attains huge spectral efficiency by removing the guard band. The channel estimation turns difficult consideringthe absolute field Orthogonality for FBMC. This paper suggests new Zadoff-chu sequence based preamble design with FBMC/OQAM to study the performances of PAPR, CCDF and BER.
The preparation of the article are as follows: Section 2 summarizes the Related Work, Section 3 examines Problem statement and the System Architecture of proposed System. Section 4 contributes the Simulation Results. The Conclusion wasconferred in Section 5.

\section{Related literatures}

Current Analysis on the $5 \mathrm{G}$ wireless communications have admitted a much healthier wave other than OFDM systems. Numerous proposals need to accept new waveforms with upgraded spectral containment. The 5GNOW project initiated in Europe, encounters challenges in LTE and LTE-Advanced [6] in handling the changing requests of $5 \mathrm{G}$. The $5 \mathrm{GNOW}$ had identified some alternateadoptions of waveforms to serve well for $5 \mathrm{G}$ requirements. These waveforms are created based on various types of filtering, among this FBMC method suits distinct needs of numerous applications. Further it has some drawbacks in frequency and timing synchronization near receiver.

Earlier, number of authors hadnoticed the issue of CFO and TO in FBMC. Despite, the proposalsconsidered are different from the effort given in this paper. While here preambles and pilots for carrier and timing acquisitions are used, almost the previous work manipulates the statistical properties of the FBMC signals, i.e., blind methods. Blind synchronization of carrier offset and timing estimation method for FBMC without knowledge of channel has been proposed by [7]. The work presented by [8] has not indicated the short preamble, but assumptions are made that CFO exists the choice of half symbol spacing. The long preamble suggestedestablish complex-valued pilot symbols that occupy all subcarriers. There are lot of dissimilarity in the long preamble proposed by [9] and the one proposed by [10] has real-value and only employs the alternate subcarriers, leaving the extra subcarriers vacant. This adaption of the 
extended preamble weredetermined through numerical results by [11] hasimproved the ability of the CFO estimates remains a major drawback and consumes more spectrum.

\section{Problem statement}

In OFDM, Orthogonality between subcarriers allows controlling ICI by ensuring time and frequency synchronization at the receiver. OFDM has very large side lobes, rectangular pulse shaping exhibit poor stop band attenuation and requires guard band at spectrum to regulate the control out-of-band emissions. OFDM uses cyclic prefix to reduce ISI, which limits the spectral efficiency. These problems are overcome by FBMC/OQAM which adopts Zadoff-chu sequence based preamble to decrease the carrier frequency and timing synchronization. The suggested method does not transmit a cyclic prefix or guard band and shapes subcarriers using well frequencylocalized waveforms that suppress signals side lobes, thus providing larger spectral capabilities.

The system complexity proposed by [12] has reduced the preamble based (or pilot based) synchronization. Zadoff-Chu sequence based preamble has been proposed to decrease the CFO and STO estimation that avoids intrinsic interference and consumed for channel estimation. In this article, a periodic Zadoff-Chu sequence preamble has been considered; both STO and CFO estimators are designed to suitleast-square approach. The fore mentioned hastime domain approach which exhibits a stable performance independently of the actual TO. This method also delivers good strength against multipath channels but has relativelymoderate complexity. By using Zadoff-Chu sequence preamble, accurate CFO has been obtained to see the reduced PAPR results and improved BER performance.

\subsection{Proposed system architecture}

The focus of the suggested architecture tries toattain better spectral efficiency adopting Zadoff-chu sequence based preamble design with FBMC/OQAM to minimize the BER. The suggested system architecture has been as shown in the Fig 1 .

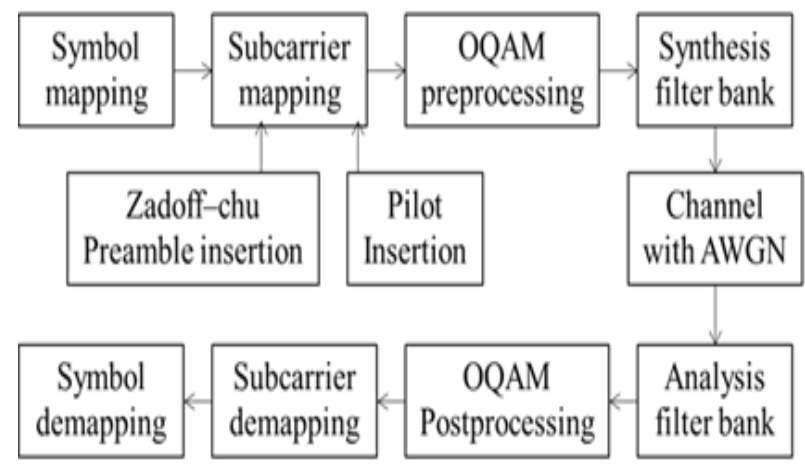

Fig. 1: Proposed Block Diagram for FBMC.

The block diagram consist of transmitter, receiver and channel with additive white Gaussian noise (AWGN). The Transmitter consists of Symbol mapping, where the digital data has been modulated using the any one of digital technique namely QPSK, 4-QAM or 64QAM and BER of the scheme was mainly found on this block. The need of subcarrier mapping would be useful for the FBMC frame creation that consists of preambles, data pilots and data subcarriers. Each FBMC frame hasprovided with a preamble particularly designed for prompt tuning of carrier frequency and timing synchronization at the receiver, upon the acknowledgement of each packet. Pilots areadopted for the efficient channel estimation and equalization that wasrequired to appreciate spectral efficiency, spectrum sharing access or high mobility scenarios. OQAM processing scheme simultaneously employs an improved pulse shaping, and interpolation by factor 2 for transmit at the Nyquist rate. The additional filtering, together with the IFFT/FFT operation and serial to parallel conversion forms a synthesis filter-bank structure, where the prototype filter was designed to significantly suppress ISI.
The ideal receiver achieves the exact adverse operation particular to transmitter. The parameters (time, frequency and phase) of transmitted FBMC signal must be observed exactly across the receiver. They are timing and frequency synchronization, channel estimation, channel equalization and phase tracking. In FBMC, Timing Offset (TO) and Carrier Frequency Offset (CFO) results in ISI (Inter-Symbol Interference) and ICI (Inter-Carrier Interference). Pilot aided and blind synchronization methods are consumed to provide the synchronization. FBMC only satisfies the Orthogonality in the real domain, [13] which causes it suffering from intrinsic interference even if perfect Timing and frequency synchronization has been achieved. However, to avoid the intrinsic interference originated from adjacent symbols in time domain, more than a couple of FBMC symbols either pilots or preambles must be allocated only for channel estimation purpose. Generally the pilots are consumed to eliminate the interference. By doing so, the received main pilots become interference-free, and channel estimation can be performed.

\subsection{Zadoff-chu sequence based preamble design}

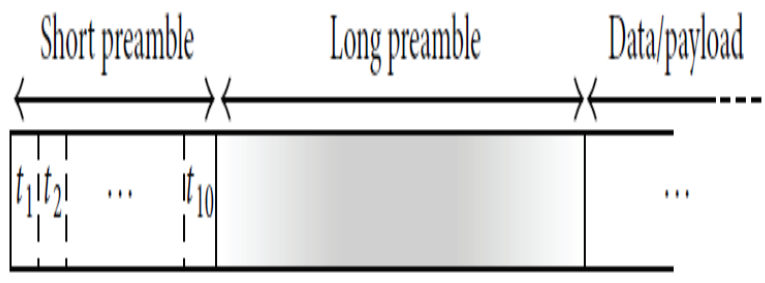

Fig. 2: FBMC Data Packet with Short and Long Preambles

Fig 2. depicts the short preamble that consists 10 cycles of a periodic signal with a summation of multiple tones of subcarrier with broaderdistance than the subcarrier spacing to acceptfinding of large CFO values. It allows to find a coarse estimation of CFO, in order that the $\mathrm{CFO}$ would be in reach of half symbol spacing. The payload encloses information equivalent to length of the frame, the data rate and the channel code. As in OFDM, the long preamble does not start with a guard interval. All coordination are retained and the receiver arequalified to accurately detect payload at the end of long preamble. The long preamble in FBMC frame arearrangedequivalently and underlying filters don'tcover the short preamble and payload of the frame.

The design considerationhas been suggested to achieve this; the quantity of the enlarged training [14] should be at least balance thelimit of prototype filter $(\mathrm{K})$ plus by a factor 2 . Hence, efficient prototype filter heads to shorter preamble and thus bandwidth efficient system was more. This has beenanalysed by using wider subcarrier that increases the bandwidth of each subcarrier;correspondingly reduce the symbol period and quantity of the prototype filter. The suggested preamble makes an attempt to control the quantity that improves the bandwidth efficiency. This development in advancement of BER and reduction in PAPR values. A lengthier preamble allows a more accurate appraisal of CFO and the key parameter for preamble design was FBMC that satisfies both conditions. The proposed Zadoff-Chu (ZC) sequence [15] has efficiently utilized for generating a long preamble that achieves superimposed $\mathrm{ZC}$ sequences used for preamble symbols.Zadoff-Chu sequences are consumed in the 3GPP LTE Long Term Evolution air interface in the Primary Synchronization Signal, uplink control channel, uplink traffic channel and sounding reference signals. Thus the proposed $\mathrm{ZC}$ sequences would be useful for random accessing of preamble in $5 \mathrm{G}$ evaluation. The proposed Zadoff-Chu sequences are an improvement over the Walsh Hadamard precoding used in universal mobile telecommunication because they effect in a constant-amplitude output signal, reducing the cost and complexity of the radio's power amplifier.

$\mathrm{ZC}$ sequence was generated by

$\mathrm{x}_{-} \mathrm{u}(\mathrm{k})=\mathrm{e}^{\wedge}(-(\mathrm{j} \pi \mathrm{uk}(\mathrm{k}+\mathrm{Nmod} 2)) / \mathrm{N}), \mathrm{k}=0,1, \ldots . \mathrm{N}-1$. 
Where $\mathrm{u}$ is the root index, there are two root indexes treated in this work "ZC_RootIndex1" and "ZC_RootIndex2". The two root indexes are allowed to generate two different sets of preamble that generates a repeated structure of preamble. The repeated structure of preamble was also useful for carrier frequency, timing synchronization and channel estimation. $\mathrm{N}$ is the length of the sequence that wasachieved by subcarriers used in the FBMC.The preamble is generated and FBMC frame is created by allowing the $\mathrm{ZC}$ sequence preamble. Now, this framecommunicates over the channel with AWGN. The parameters of frame get affected by noise, the receiver desires to find the noise and generate the accurate results. Thus, the $\mathrm{ZC}$ preamble is useful in receiver section.

\subsection{Carrier frequency offset and timing offset synchro- nization}

The carrier frequency offset has been achieved by angle of the correlation peak and the timing offset synchronization[6] has been achieved by the cross-correlation between local proposed ZC sequences and received preamble sequences. The searching range was one frame length, so the maximum delay of the input signal should not exceed one frame length. The peaks selected for estimation was chosen by filter coefficients and the number of preamble symbols per frame and the interval between two filter tap was the time that a preamble symbol last.

\subsection{Channel estimation}

The suggested preamble symbol [16]has been preferred to do channel estimation thatallows the generation of pilot sequence. The value of preamble symbols in transmitter was known and the repeated structure formed cyclic prefix in preamble that make it robust to multipath. The least-squares channel response estimate at subcarrier ' $\mathrm{i}$ ' can be attained as: $\mathrm{H}_{\mathrm{i}}=\mathrm{Y}_{\mathrm{i}} / \mathrm{X}_{\mathrm{i}}$, where $\mathrm{Y}_{\mathrm{i}}$ is the received symbol and $\mathrm{X}_{\mathrm{i}}$ is the transmitted preamble symbol on the $\mathrm{i}^{\text {th }}$ subcarrier.

\subsection{Phase tracking}

The compensated phase estimates by pairs of pilots and ZC sequence preambles in frame. The tracking aspirescalculating a channel estimate by allowing transmitter and receiver data on the pilots at two different time instants. The phase was calculated as mean value of the angles of pilots and preambles.

\section{Simulation results}

The proposed work uses the following simulation parameters as shown in Table 1. for the performance measures such as Complementary Cumulative Distribution Curve (CCDF) for FBMC signal with different multicarrier to analyse the Peak to Average Power Ratio (PAPR) and BER, MSE for spectral analysis. The frequency offset and timing offset with respect to MSE was also considered.

\begin{tabular}{ll}
\multicolumn{2}{c}{ Table 1: Simulation Parameters } \\
\hline Modulation Type & QPSK or 4-QAM \\
\hline Number of Sub Carriers & $128(120$ for Data carriers, \\
Sampling Rate & 8 for Pilot carriers $)$ \\
Carrier Signal Power $(1 \mathrm{GHz})$ & $20 \mathrm{MHz}$ \\
Prototype Filter & $0.01 \mathrm{w}$ \\
Filter Overlapping Factor & $\mathrm{PHYDYAS}$ \\
FBMC Root Index 1 & $\mathrm{P}[2]=1 / \sqrt{2, \mathrm{P}[3]=0.235)})=0.9715983$, \\
FBMC Root Index 2 & 7 \\
\hline
\end{tabular}

The efficiency of Frequency over Spectrum power is shown in Fig. 3 . The OFDM utilizes the frequency spectrum of $10 \mathrm{MHz}$ bandwidth out of which $9 \mathrm{MHz}$ has been utilized for subcarrier transmission and remaining $1 \mathrm{MHz}$ was utilized for guard band which wastes the spectrum. To overcome this issue, different multi carrier techniques such as F-OFDM, GFDM, UFMC and FBMC for 5G spectrum was analysed. The observation shows that the FBMC technique enhancement was more than the other multi carrier techniques anticipated the efficient usage of spectrum. The suggested FBMC method efficiently suppresses the side lobes and reduces out - of - band emission. It was realised that $\mathrm{FBMC}$ with well-designed preambles and prototype filters helps to enhance the spectrum of each sub carriers within limited bandwidth.

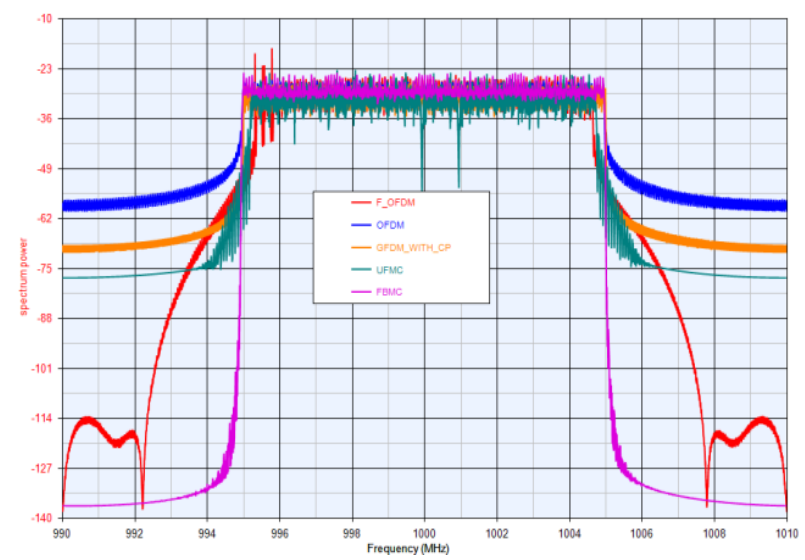

Fig. 3: Frequency over Spectrum Power.

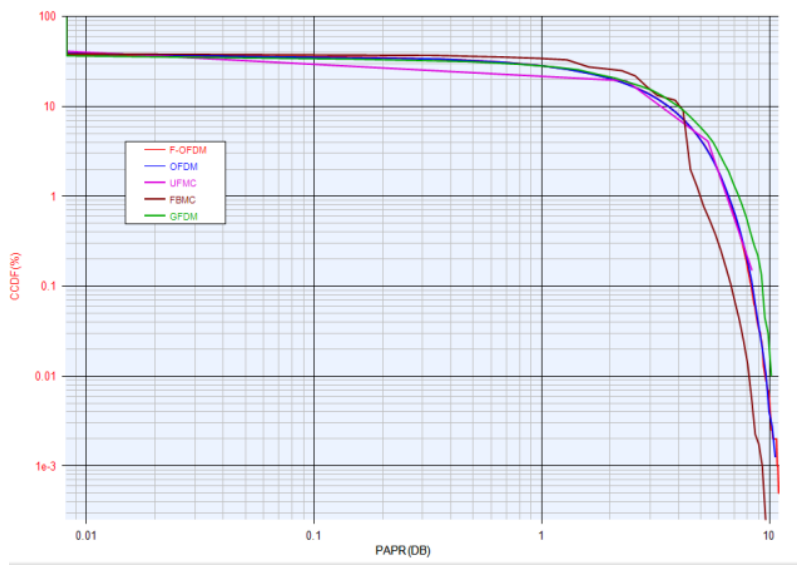

Fig. 4: PAPR over CCDF.

The efficiency of PAPR over CCDF is shown in Fig.4. PAPR was the common metric to characterize the amplitude fluctuations of the signal. These amplitude fluctuations results in out of band emissions, spectrum re-growth, causing ICI and ISI. The CCDF curve shows according to what time the signal spends at or above a given power level. The PAPR value of FBMC has been reduced to $2 \mathrm{~dB}$ for $1 \%$ of $\mathrm{CCDF}$ while correlated with other access techniques of $5 \mathrm{G}$. The F-OFDM and UFMC has the same PAPR values of OFDM but GFDM has more PAPR values than OFDM which becomes a drawback for GFDM.

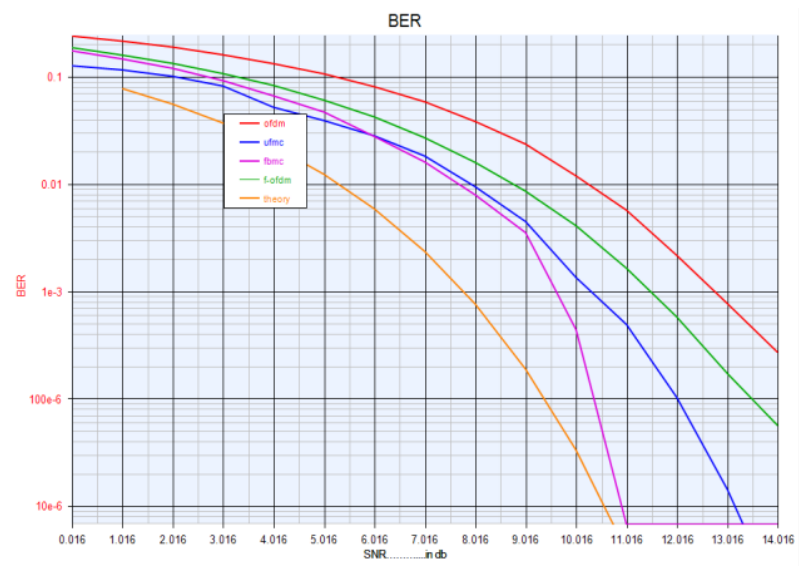

Fig. 5: SNR over BER. 
The achievement of SNR over BER is shown in Fig.5. The BER response of FBMC was much better than OFDM and UFMC. At 1e4 of BER, the SNR of FBMC was decreased by an amount of 1.6 $\mathrm{dB}$ to UFMC, $3 \mathrm{~dB}$ to F-OFDM and $5.5 \mathrm{~dB}$ to OFDM. It was realised that the FBMC BER response has been nearer to the theoretical response such as QPSK BER.

The realization of SNR over MSE is shown in Fig.6.The intrinsic interference originated from neighbouring symbols in time domain seriously damages the pilot signal in the channel estimation stage leads to low estimation accuracy. So to achieve the channel estimation, the Mean Square Error (MSE) as a function of SNR founds to be useful. The FBMC scheme has almost no loss in most SNR ranges while OFDM scheme has a very big loss at all SNR points. Approximately, $6 \mathrm{~dB}$ gain could be attained by FBMC over the OFDM. Such improvement was appropriate to the efficient interference utilization property.

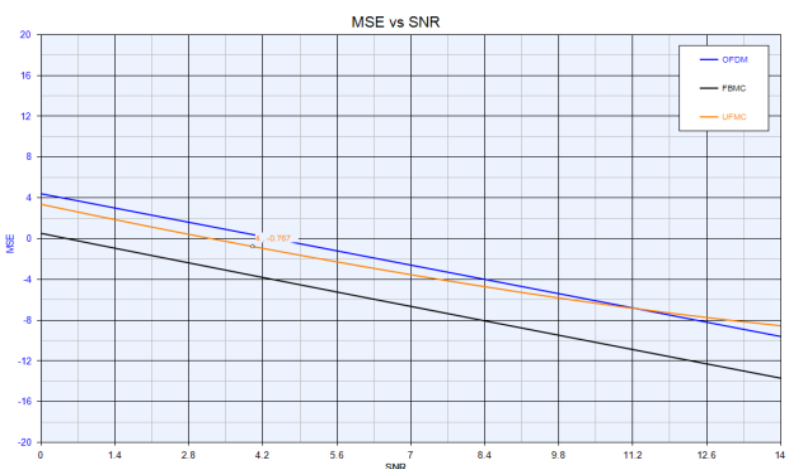

Fig. 6: SNR over MSE.

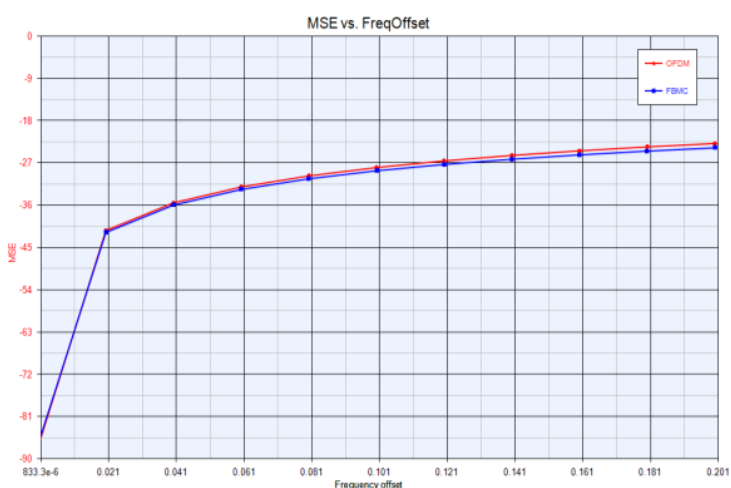

Fig.7: Frequency Offset over MSE.

The performance of Frequency offset over MSE is shown in Fig.7. In OFDM, inadequate frequency domain synchronization with different uplink users leads to MSE affected by inter-carrier interference (ICI). In FBMC, even a small CFO affects deficiency of Orthogonality among the different subcarriers and thus introduces ICI. Furthermore, carrier frequency offset (CFO) results in ISI in a multi carrier OQAM system. The graph presents mean square error (MSE) or distortion in charge of ICI, ISI at the receiver. Based on the observation, FBMC has $1.2 \mathrm{~dB}$ less distortion than OFDM during the normalized carrier offset. As a result, if a system requires high SNR, FBMC outperforms than OFDM in terms of CFO immunity. This indeed was an influential gain in a system with high data rate when higher order modulations are required.

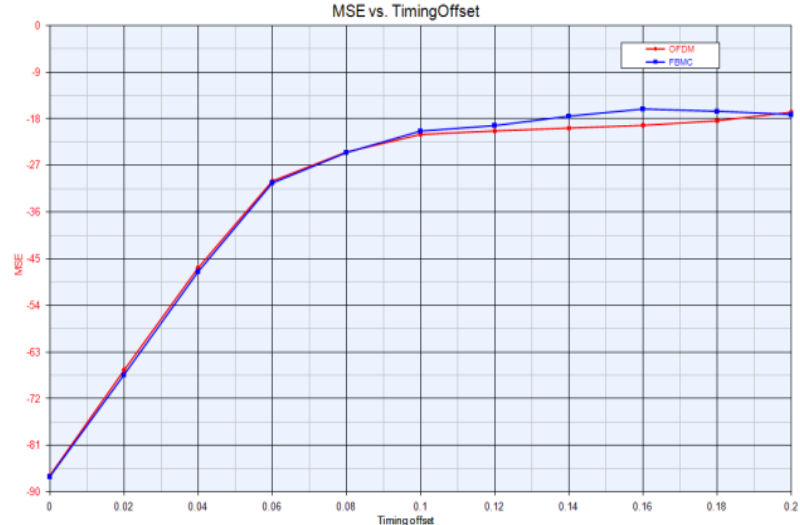

Fig. 8: Timing Offset over MSE.

The performance of Timing offset over MSE is shown in Fig.8. The number of multiuser spectral leakage due to STO for any multicarrier waveform is closely associated to the discontinuities in the observation window at the receiver. Moreover, filtering at both transmitter and receiver sides benefits to lighten the performance loss caused by the timing offset. It is realised that FBMC has $0.2 \mathrm{~dB}$ less distortion than OFDM at the timing offset. The suggested preamble base FBMC scheme has performed better than conventional multicarrier scheme. Therefore, it was crucial to have accurate timing offset estimation methods when higher SNRs are required.

\section{Conclusion}

This framework suggests a new preamble design and corresponding channel estimation algorithm for FBMC/OQAM system. The Zadoff-chu sequence were allowed to generate the long preamble structure for the frame. The performance results demonstrates that the suggested preamble based method performs well than the conventional preamble structure in attributes of spectral efficiency, and reduced PAPR values. Moreover, the suggested algorithm has low complexity that makes efficient BER performance with respect to SNR and MSE for corresponding frequency offset and timing offset. Hence it manages forapplying directly to advanced mobile systems like $5 \mathrm{G}$. This work may be extended to MIMO FBMC in future because it offers many exciting problems for research.

\section{References}

[1] Behrouz Farhang-Boroujeny, OFDM versus Filter Bank Multicarrier, IEEE Signal Processing Magazine, 2011,Vol. 28, pp. 92-112.

[2] Naser Al-Falahy, Omar Y. Alani, Technologies for 5G Networks: Challenges and Opportunities, 2017, IEEE IT Pro, Vol: 19, Issue: 1, pp.12-20.

[3] Behrouz Farhang-Boroujeny, Filter Bank Multicarrier Modulation: Waveform Candidate for 5G and Beyond, 2015, Hindawi Publishing Corporation Advances in Electrical Engineering,Vol 2014.

[4] Nan Tang, Shiwen He, ChunlinXue, Yongming Huang, Luxi Yang, IQ Imbalance Compensation for Generalized Frequency Division Multiplexing Systems, 2017, IEEE Wireless Communications Letters, Vol. 6, No. 4, pp.422-425.

[5] A.Viholainen, T.Ihalainen, T. Hidalgo Stitz, M.Renfors, and M.Bellanger,Prototype filter design for Filter bank based Multicarrier Transmission, 2009in Proc. European Signal Processing Conf., Glasgow, Scotland, pp. 1359-1363

[6] Juho Lee, Younsun Kim, YongjunKwak, Jianzhong Zhang, ArisPapasakellariou, Thomas Novlan,Chengjun Sun, and Yingyang Li, LTE-Advanced in 3GPP Rel -13/14: An Evolution Towards 5G, 2016,IEEE Communications Magazine Vol. 54, Issue: 3.

[7] Tilde Fusco, AngeloPetrella and Mario Tanda, Data-aided symbol timing and CFO synchronization for filter bank multicarrier systems, 2009, IEEE Transactions on Wireless Communications, Vol: 8, Issue: 5, pp. 2705-2715.

[8] Jeong-Min Choi, Youngho Oh, Hakju Lee, and Jong-SooSeo, Interference-dependent pair of pilots for channel estimation in FBMC systems, 2016 in IEEE International Symposium on Broadband Multimedia Systems and Broadcasting, pp. 1-4. 
[9] Stijn Van Caekenberghe, André Bourdoux, and JérômeLouveaux, Preamble based frequency-domain joint CFO and STO estimation for OQAM-based filter bank multicarrier, 2014, EURASIP Journal on Advances in Signal Processing.

[10] Behrouz Farhang-Boroujeny and P. Amini, Packet format design and decision directed Tracking methods for Filter bank Multicarrier Systems, EURASIP Journal on Advances in Signal Processing, 2010, Vol.2010, Article ID 30798.

[11] Quang Duong, Ha H. Nguyen, Walsh-Hadamard Precoded Circular Filter bank Multicarrier Communications, 2017, International Conference on Recent Advances in Signal Processing, Telecommunications \& Computing (SigTelCom), pp. 193 - 198.

[12] Jamal Bazzi, Petra Weitkemper, and KatsutoshiKusume, Power efficient scattered pilot channel estimation for FBMC/OQAM, in 10th International ITG Conference on Systems, Communications and Coding, 2015, pp. 1-6.

[13] H.Saeedi-Sourck, S.Sadri,Y.Wu and B.Farhang-Boroujeny, Near maximum likelihood synchronization for filter bank multicarrier systems, 2013, IEEE Wireless Communications Letters, Vol: 2, Issue: 2, pp. 235-238.

[14] Esteban Gutiérrez, José A López-Salcedo and Gonzalo Seco-Granados, Systematic design of transmitter and receiver architectures for flexible filter bank multi-carrier signals, EURASIP Journal on Advances in Signal Processing, 2014, Vol. 103, Issue: 1, pp.1-26.

[15] Branislav M. Popović, Efficient DFT of Zadoff-Chu sequences, 2010, Electronics Letters, Vol. 46, No. 7, pp. 502-503.

[16] Jinfeng Du and Svante Signell, Novel preamble-based channel estimation for OFDM/OQAM system, 2009, IEEE ICC. 